\title{
Assessment of Extent and Severity of Coronary Artery Stenosis Using CT Coronary Angiography Among Prediabetic Patients \\ Eman Elkholy ${ }^{1 *}$, Layla Ahmed ${ }^{2}$, Rehab M. Hamdy², Ahmed Magdy ${ }^{3}$ \\ ${ }^{1}$ Mahalla Cardiac Center, ${ }^{2}$ Department of Cardiology, Faculty of Medicine (for Girls), Al-Azhar University, Cairo, Egypt, ${ }^{3}$ Kobry El-Kobba Military Hospital \\ *Corresponding author: Eman Elkholy, Mobile: (+20)01068343683, E-mail: emanelkholy23@gmail.com
}

\begin{abstract}
Background and aims: Prediabetes is associated with an increased risk of developing diabetes and cardiovascular disease. Our objective was to assess the extent and severity of coronary artery diseases (CAD) among prediabetic patients using multi-slice computed tomography (MSCT) coronary angiography.

Subjects and methods: Our population included 60 patients with mean age $56.2 \pm 10.5$ years presented by typical chest pain with fasting blood glucose (FPG) $<126 \mathrm{mg} / \mathrm{dL}$ and HbAlc $\leq 6.4 \%$ and at least one CV risk factor. Any patients with prior history of CV disease or clinical evidence of advanced renal disease were excluded from this study. The extent and severity of coronary artery stenosis were assessed using MSCT coronary angiography. The patients were divided into two groups, prediabetic group (GI) and non-diabetic group (GII). Prediabetes was defined as an HbA1c value of 5.7-6.4\%.

Results: Prediabetic group had significantly higher number of diseased vessels with higher number of patients having MVD compared to non-diabetic group. HbA1C and FPG were found as independent predictors for both extent and severity of diseased coronary arteries.

Conclusion: $\mathrm{CAD}$ was more advanced in prediabetic patients compared to non-diabetic patients. Moreover, non-diabetic levels of glucose metabolism evidenced by impaired FBG and increased levels of HbA1C were helpful to predict increased risk for the extent and severity of CAD among our study population.
\end{abstract}

Keywords: Prediabetes, HbA1c, coronary artery disease, MSCT coronary angiography.

\section{INTRODUCTION}

Prediabetes is a metabolic stage intermediate between normal glucose homeostasis and diabetes ${ }^{(1)}$ and is associated with an increased risk of developing diabetes and cardiovascular disease ${ }^{(2)}$.

The American Diabetes Association (ADA) proposed that glycated hemoglobin A1c (HbA1c) could be used as a diagnostic test for diabetes and prediabetes as an alternative to impaired fasting glucose (IFG), or impaired glucose tolerance (IGT) ${ }^{(3)}$.

An HbA1c value of (5.7-6.4\%) was recommended for the diagnosis of prediabetes. Increasing levels of $\mathrm{HbA} 1 \mathrm{c}$ in individuals without DM are associated with the presence and burden of coronary atherosclerosis and mixed coronary arterial plaques ${ }^{(4)}$.

There are several methods of screening for cardiovascular disease, among these, coronary artery calcium (CAC) score by coronary computed tomography $(\mathrm{CCT})^{(5)}$.

Coronary angiography is the gold standard for diagnosis of coronary artery disease, but its invasiveness nature prevents its regular use in asymptomatic patients.

Coronary artery calcium (CAC) score is a strong predictor of incident coronary heart disease ${ }^{(\boldsymbol{\sigma})}$ and is considered as one of the best subclinical coronary atherosclerosis markers ${ }^{(7)}$, especially in asymptomatic individuals at intermediate cardiovascular risk ${ }^{\left({ }^{(8)}\right.}$.
MSCT is a non-invasive technology to visualize the coronary arteries and found to be safe and reliable to detect or exclude coronary artery disease (CAD).

MSCT appears to be useful as a first-line imaging technique in carefully selected patients to evaluate the need of an invasive coronary angiography ${ }^{(9)}$.

\section{AIM OF THE WORK}

Our objective was to assess the extent and severity of coronary artery diseases among prediabetic patients using multi-slice computed tomography (MSCT) coronary angiography.

\section{PATIENTS AND METHODS}

\section{Study population:}

This study was conducted on 60 patients having typical chest pain. The patients were selected from those attending cardiology outpatient clinic at Kobri El-Kobba Military Hospital and referred for multislice CT angiography (MS-CT), for diagnosis of coronary artery disease. The study was conducted during the period from June 2018 to December 2018. Inclusion criteria were; patients with typical chest pain and having: $\mathrm{FBG}<126 \mathrm{mg} / \mathrm{dL}, \mathrm{HbA} 1 \mathrm{c} \leq 6.4 \%$ and at least one cardiovascular $(\mathrm{CV})$ risk factor among the following: hypertension, dyslipidemia, family history of IHD or premature sudden cardiac death or current smoking.

Arterial hypertension was considered present when measurement of brachial blood pressure (BP) 
exceeded $140 \mathrm{mmHg}$ (systolic) and/or $90 \mathrm{mmHg}$ (diastolic) on at least two different occasions, or if the patient was on antihypertensive medication. Dyslipidemia was defined as a total serum cholesterol level of $>190 \mathrm{mg} / \mathrm{dL}$, serum low-density lipoprotein (LDL) cholesterol level of $>115 \mathrm{mg} / \mathrm{dL}$, serum high density lipoprotein (HDL) cholesterol level of $<40$ $\mathrm{mg} / \mathrm{dL}$ for men and $<45 \mathrm{mg} / \mathrm{dL}$ for women, or serum triglycerides level of $>150 \mathrm{mg} / \mathrm{dL}$, or history of daily intake of any lipid-lowering medication. Patients with prior history of diabetes, known CV disease or advanced renal disease were excluded.

\section{Ethical approval:}

The study protocol approved by the medical Ethics Committee of the Faculty of Medicine for Girls, AlAzhar University and a verbal informed consent was obtained from all participants before enrollment into the study.

\section{Laboratory investigations, ECG and echocardiography}

All patients were subjected to routine clinical examination. Fasting blood samples were collected to ascertain levels of glucose, total cholesterol, triglycerides, LDL and HDL cholesterol, HbA1C and creatinine.

12-leads surface ECG was done to exclude any ECG changes suggesting ischemia as ST-T wave changes or pathological $\mathrm{Q}$ wave of prior myocardial infarction. Echocardiography was performed to asses LV dimensions, functions, LV resting wall motion abnormalities.

\section{Multi-slice CT angiography:}

The CT angiography was performed to all patients utilizing a dual source scanner (Somatom Definition Flash) using two X-ray sources for better visualization of the coronary tree at relatively high heart rates and all coronary arteries were evaluated at different phases of the cardiac cycle by acquisition of thin slice sections (0.6 mm).
All scans were preceded by non-contrast enhanced scan for coronary artery calcium (CAC) score (sequential scan with $32 \times 0.6 \mathrm{~mm}$ collimation, tube current $60 \mathrm{mAs}$ at $120 \mathrm{kV}$ ) to rule out patients with dense coronary calcification (total score above 1000 Agatston score). Coronary segments were visually scored for the presence of coronary plaque using segment involvement score (SIS) and segment stenosis score (SSS).

The included patients were divided into two groups according to American Diabetes Association (ADA) criteria ${ }^{(10)}$ based on plasma levels of FPG and HbA1c; prediabetic group: (FPG $100-125 \mathrm{mg} / \mathrm{dL}$ and HbA1c $5.7-6.4 \%$ ) and non-diabetic group (FPG < $100 \mathrm{mg} / \mathrm{dL}$ and $\mathrm{HbA} 1 \mathrm{c}<5.7 \%$ ).

\section{Statistical analysis}

All analyses were performed using SPSS for Windows (version 23.0, SPSS, Chicago, Illinois, USA). Kolmogorov-smirnov test was used to discriminate between normal and non-normal distributed variables. Descriptive statistics were presented using mean and standard deviation (SD) for normally distributed quantitative variables, and the median and interquartile range (IQR) for non-normally distributed variables. Comparisons of parametric values between 2 groups were performed by the student $t$ test and comparisons for non-parametric values between 2 groups were performed by MannWhitney U test. Categorical variables were compared with the chi square test. A two-tailed $P<0.05$ was considered significant. Correlation and Multi-variate logistic regression used to identify independent predictors of extent and severity of coronary artery disease.

\section{RESULTS}

The baseline characteristics of the study subjects presented in table 1. Prediabetic subjects were older than the non-diabetic but other risk factors were equally distributed between the 2 groups.

Table 1: Demographic characteristics of pre-diabetic and non-diabetic groups

\begin{tabular}{|l|l|l|l|l|}
\hline \multicolumn{2}{|c|}{ Variables } & $\begin{array}{c}\text { Group I } \\
\text { N=30 }\end{array}$ & \multicolumn{1}{c|}{$\begin{array}{c}\text { Group II } \\
\text { N=30 }\end{array}$} & p value \\
\hline Age (years) & $59.1 \pm 7.86$ & $53.4 \pm 12$ & $\mathbf{0 . 0 3}$ \\
\hline \multirow{2}{*}{ Gender } & Male & 28 & 27 & $\mathbf{0 . 6 4}$ \\
\cline { 2 - 5 } & Female & 2 & 3 & $\mathbf{0 . 2 9 7}$ \\
\hline HTN & 15 & 11 & $\mathbf{0 . 7 9 3}$ \\
\hline Smoking & 18 & 17 & $\mathbf{0 . 6 0 2}$ \\
\hline F Hx of IHD & 14 & 12 & \\
\hline
\end{tabular}

Laboratory measurements of the patients stratified into 2 groups shown in table 2. Prediabetic group had significantly higher value of $\mathrm{HbA1C}$ and FBG compared to non-diabetic group. However, no significant difference could be detected between both groups regarding serum creatinine or lipid profile. 
Table 2: Laboratory investigation among prediabetic and non-diabetic groups

\begin{tabular}{|l|c|l|l|}
\hline \multicolumn{1}{|c|}{ Variables } & $\begin{array}{c}\text { Group I } \\
\text { N=30 }\end{array}$ & $\begin{array}{c}\text { Group II } \\
\text { N=30 }\end{array}$ & P value \\
\hline S.Cr (mg/dL)Mean \pm SD & $0.952 \pm 0.184$ & $0.955 \pm 0.215$ & 0.954 \\
\hline HbA1C (\%) Mean \pm SD & $5.99 \pm 0.21$ & $5.06 \pm 0.37$ & 0.0001 \\
\hline FBG (mg/dl) Mean \pm SD & $113 \pm 7.28$ & $84.8 \pm 8.79$ & 0.0001 \\
\hline Cholesterol $(\mathbf{m g} / \mathbf{d l})$ Mean \pm SD & $214.2 \pm 7.9$ & $210.4 \pm 7.4$ & 0.843 \\
\hline TG (mg/dl) Mean \pm SD & $173 \pm 7.3$ & $155 \pm 8.8$ & 0.361 \\
\hline HDL (mg/dl) Mean \pm SD & $44.1 \pm 11.2$ & $46.6 \pm 8.58$ & 0.335 \\
\hline LDL (mg/dl) Mean \pm SD & $126 \pm 7.8$ & $130 \pm 33.8$ & 0.709 \\
\hline
\end{tabular}

The median of CAC score was higher in the prediabetes group compared to median of non-diabetic group as shown in table 3.

Prediabetic group had significantly higher number of diseased vessel detected by CT coronary angiography compared to non-diabetic group. In addition, prediabetic group had significantly higher number of patients with multi-vessel disease (MVD) while non-diabetic group had significantly higher number of patients with single vessel disease. There was statistically significant difference between both groups regarding the severity of lesion as shown in table 3. The most common affected coronary artery in both groups was LAD (either single or in MVD)

Table 3: Comparison between prediabetic and non-diabetic groups regarding CT- coronary angiographic data

\begin{tabular}{|c|l|l|c|l|}
\hline \multicolumn{2}{|l|}{ Variables } & $\begin{array}{c}\text { Group I } \\
\mathbf{N = 3 0}\end{array}$ & $\begin{array}{c}\text { Group II } \\
\mathbf{N = 3 0}\end{array}$ & P value \\
\hline Ca Score (AU) Median \pm IQR & $\begin{array}{c}106.100 \\
(0-235.225)\end{array}$ & $\begin{array}{c}0.000 \\
(0-53.725)\end{array}$ & 0.007 \\
\hline \multirow{3}{*}{ No of Vs affected } & No CAD & 7 & 13 & 0.100 \\
\cline { 2 - 5 } & One vessel & 6 & 12 & 0.091 \\
\cline { 2 - 5 } & Two vessels & 5 & 3 & 0.448 \\
\cline { 2 - 5 } & Multi-vessel disease & 12 & 2 & 0.002 \\
\hline \multirow{3}{*}{$\begin{array}{c}\text { Severity of } \\
\text { coronary lesion }\end{array}$} & Total occlusion & 6 & 1 & 0.044 \\
\cline { 2 - 5 } & Significant stenosis & 16 & 8 & 0.035 \\
\cline { 2 - 5 } & $\begin{array}{l}\text { Non-significant } \\
\text { stenosis }\end{array}$ & 1 & 8 & 0.011 \\
\hline
\end{tabular}

This study showed that there were significant positive correlation between both extent and severity of affected coronaries with HbA1C, FBG, total cholesterol, triglycerides, LDL-cholesterol, and CAC score, while there was a significant negative correlation between LVEF with the extent and severity of affected coronaries lesions. By applying multi-variate logistic regression, we found that $\mathrm{HbA} 1 \mathrm{C}$ and FPG were independent predictors for both extent and severity of diseased coronary arteries among the studied population (Figure 1 and 2). Figure 3 showed MS-CT coronary angiography images from the studied population.
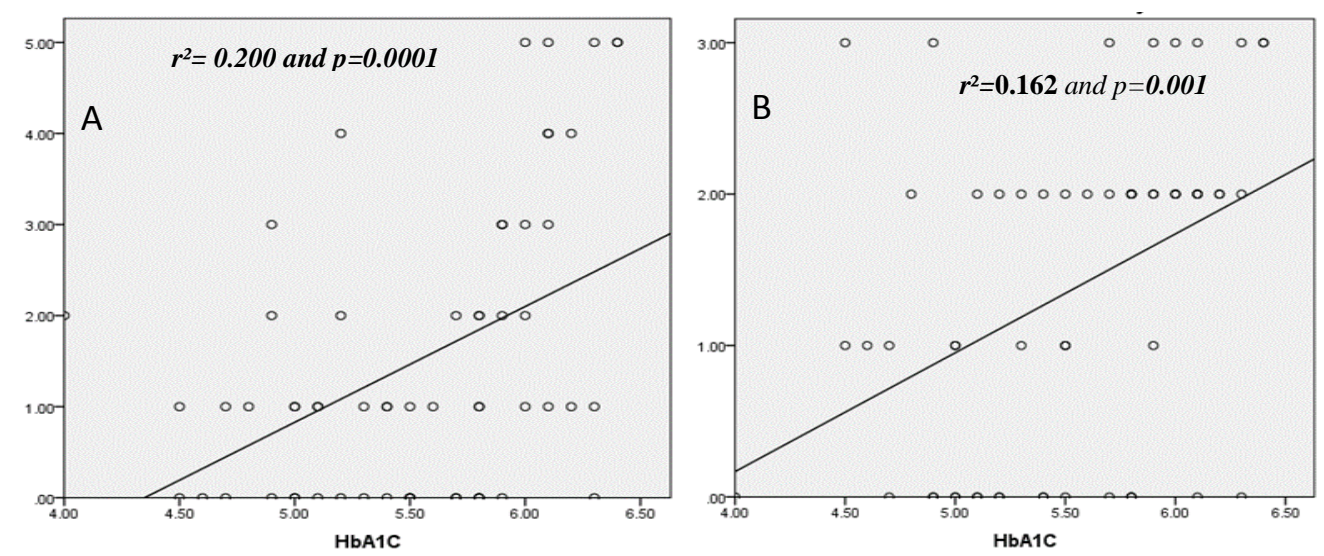

Figure (1): HbA1c as independent predictor of (A) number and (B) severity of diseased vessels among the studied population 

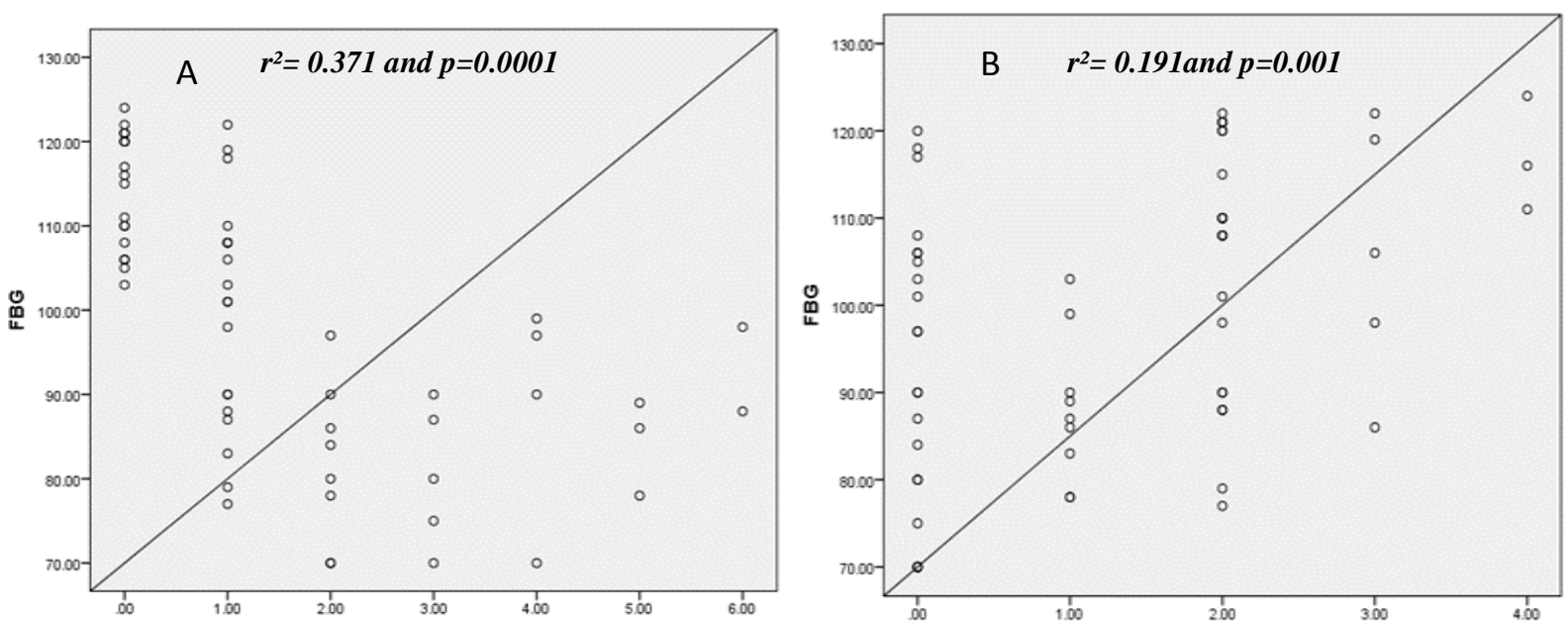

Figure (2): FPG as independent predictor of (A) number and (B) severity of diseased vessels among the studied population
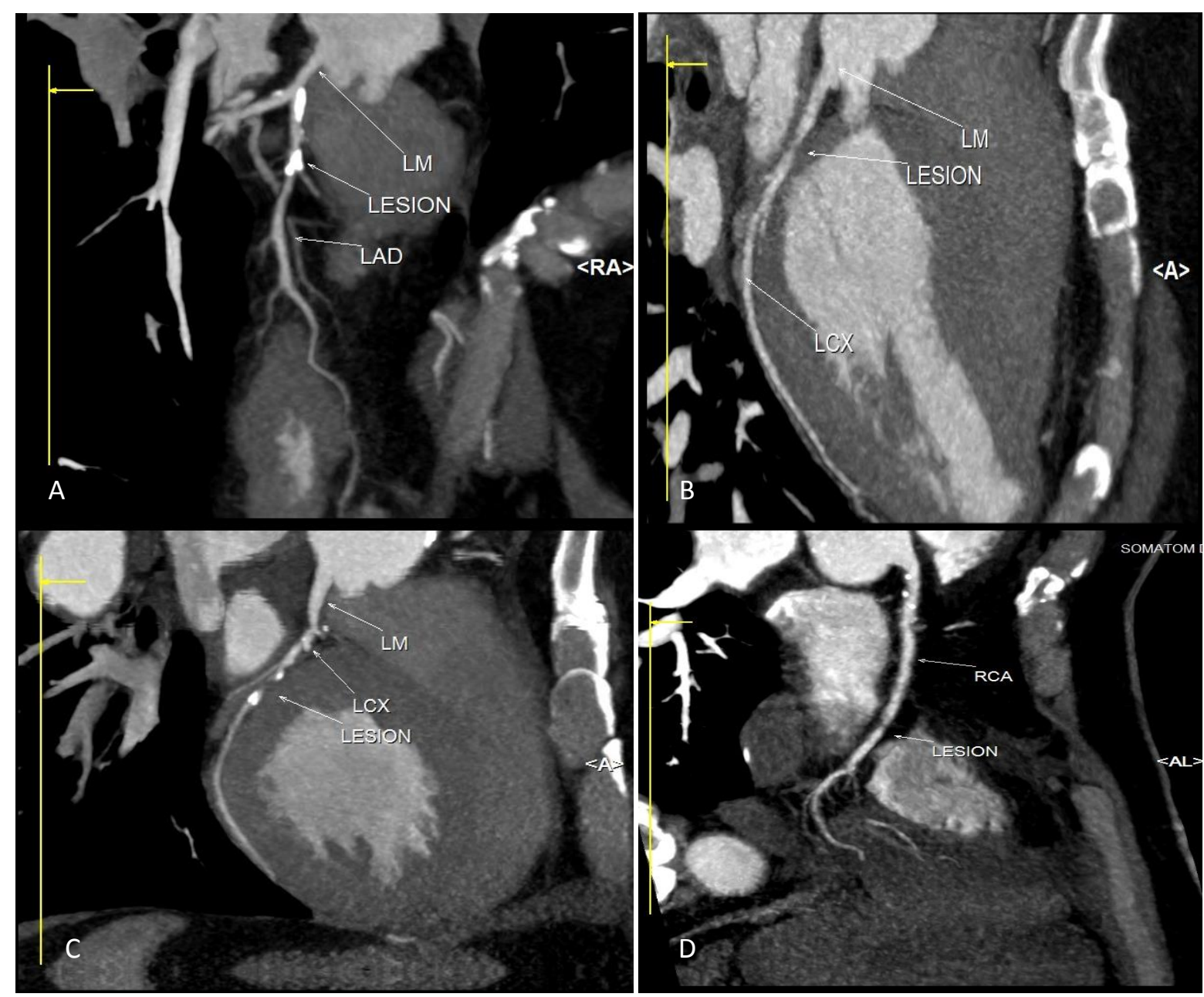

Figure (3): MS-CT coronary angiography images (A) Multi-planar reformation image showing mid segment LAD 70-80\% lesion (B) Curved multi-planar reformation image showing proximal LCX tight lesion, (C) Multi-planar reformation image showing non dominant LCX showing osteal and mid segment significant lesion and (D) Multi-planar reformation image showing co-dominant atherosclerotic vessel showing mid segment significant lesion. 


\section{DISCUSSION}

Patients with impaired fasting glucose (IFG)

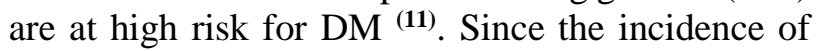
macrovascular complications is only weakly associated with the duration of diabetes, one may speculate that impaired glucose homeostasis and increasing atherogenicity occur prior to a clinical diagnosis of DM ${ }^{(12)}$. To better manage patients at high risk for future cardiac events, the identification of subclinical atherosclerosis in patients with prediabetic status has important implications.

Impaired glucose tolerance and impaired fasting glucose emerged as independent risk factors for cardiovascular disease mortality. Some reports indicated a correlation between glucose metabolism and coronary artery disease (CAD) even in patients without diabetes or IGT ${ }^{(\mathbf{1 3})}$.

However, much debate emerged regarding the better metabolic predictors of CAD in nondiabetic patients and if there is a correlation between their values and the severity of coronary artery disease $^{(14)}$.

Glycosylated hemoglobin A1c (HbA1c) is a marker of long-term glycemic control and elevated $\mathrm{HbAlc}$ was associated with an increased risk of cardiovascular diseases in patients with diabetes, moreover $\mathrm{HbA} 1 \mathrm{c}$ is also associated with all-cause of death and cardiovascular disease even in absence of diabetes $^{(15)}$.

In our study, we found that the CAC score was significantly higher in the prediabetic group than the non-diabetic group. This result was consistent with Scicali $\boldsymbol{e t}$ al. ${ }^{(1)}$ who reported that prediabetic group defined by $\mathrm{HbA} 1 \mathrm{c}$ exhibited higher CAC score compared to non-diabetic one. Furthermore, they demonstrated that $\mathrm{HbA1c}$ was an independent correlate of CAC score. Also our results is matched with the study done by Lim et $\boldsymbol{a l}{ }^{\left({ }^{(16)}\right.}$ who concluded that CAC score was higher in subjects with impaired glucose homeostasis than in norm-glycemic individuals.

In the present study, we found that prediabetic group had higher number of diseased vessel, higher multivessel disease and more total occlusions than non-diabetic group. These results are in concordant with Garg et al. ${ }^{(17)}$ who found that patients with impaired glucose tolerance (i.e, HbA1c 5.7 - $6.4 \%$ ) had markedly higher risk of $\mathrm{CAD}$, higher mean number of diseased vessels, higher left main and/or triple vessel disease, more chronic total occlusions compared to patients with $\mathrm{HbA1C}<5.7 \%$. Our findings are in consistent with the study done by Kurihara et al. ${ }^{(\mathbf{1 8})}$, who reported that both the degree of coronary atherosclerosis and the plaque vulnerability were more advanced in patients with prediabetes than in those without diabetes. Also our results are in accordance with the study done by Lim et al. ${ }^{(16)}$ who demonstrated that in terms of stenosis of the coronary arteries, subjects with IFG had more significantly stenotic coronary artery disease than did subjects with normal fasting glucose. In terms of the location of the involved artery, the current study revealed that subjects with IFG had higher incidence of diseased left main or LAD arteries than did subjects with NFG. Kurihara et al. ${ }^{(19)}$ reported that both coronary atherosclerosis and plaque vulnerability were more advanced in prediabetic patients than in those without DM and were comparable to patients with DM. Ertan et al. (20) found that the early detection of prediabetes might be associated with more coronary lesions for percutaneous or surgical revascularization and identified people at highest risk of developing overt $\mathrm{DM}$ and further advancing arteriosclerosis.

In the current study, we found that there was a significant positive correlation between FBG with extent and severity of diseased coronary arteries. Also, FBG was found as an independent predictors of both number and severity of diseased coronary arteries. Our results are similar to results of Gui et al. (21) who reported that as FPG levels increased, the number of diseased vessels, the average number of diseased vessels, and the proportion of totally occluded vessel also increased. Coutinho et al. ${ }^{(22)}$, conducted meta-regression analysis of the published data from 20 studies of 95783 individuals, who had 3707 cardiovascular events over 12.4 years, and demonstrated that fasting and post challenge glucose level of 6.1 and $7.8 \mathrm{mmol} / \mathrm{L}$ was associated with relatively higher cardiovascular risks as compared with glucose level of $4.2 \mathrm{mmol} / \mathrm{L}$. This study demonstrated progressive relation between glucose levels and cardiovascular risks extending below the diabetic threshold. However, Yan et al. ${ }^{(23)}$ found that the prevalence of significant CAD did not differ significantly between NGT and IFG subjects according to the ADA diabetes criteria 2003, which was not consistent with our findings. The discrepant results attributed to the following (1) we used ADA diabetes criteria 2010 while Yan et al. ${ }^{(23)}$ used ADA diabetes criteria 2003 with different cutoff value of FBG and (2) Yan et al. ${ }^{(23)}$ conducted the study on a selective basis, as subjects were not randomly chosen but enrolled only from hospitals with clusters of highrisk patients. Therefore, the actual prevalence of CAD might be overestimated.

In our study, we found that a significant positive correlation between $\mathrm{HbA1C}$ with number and severity of affected vessels. In addition, HbA1C was found as an independent predictor of number and severity of diseased coronary arteries. These results are consistent with the study done by Tomizawa $\boldsymbol{e t}$ 
al. (24) who noted that not only diabetic patients (HbA1c > 6.5\%\%) but also borderline patients (HbA1c 6.1-6.4) were related with extensive disease. The association of $\mathrm{HbA} 1 \mathrm{c}$ elevation and extensive disease was quite similar between borderline and diabetic patients. In a recent analysis from the Atherosclerosis Risk in Communities (ARIC) study, Selvin et al. ${ }^{(25)}$ found that risk of coronary artery disease increased in non-diabetic individuals with high baseline HbA1c.

Our findings are concordant with the study done by Garg et al. ${ }^{(17)}$, their results implied that HbA1C not only was a marker of chronic dysglycemia but also could be used as an independent predictor of CAD and its severity even in nondiabetic individuals. Measurements of $\mathrm{HbA} 1 \mathrm{C}$ levels might improve risk assessment in non-diabetic patients. Eskesen et al. (26) had demonstrated that $\mathrm{HbA1c}$ level was highly correlated with development of CVD amongst individuals without DM. Furthermore, they found a strong association between level of $\mathrm{HbAlc}$, even within the normal range and the development of DM. Our findings are consistent with the study done by Kurihara et al. ${ }^{\left({ }^{(19)}\right.}$, who reported that coronary atherosclerosis and plaque vulnerability were more advanced in prediabetic than in nondiabetic patients and comparable between prediabetic and diabetic patients. Slight or mild disorders in glucose metabolism, such as prediabetes, could be a risk factor for CAD, as is diabetes itself. Ayhan et al. (27) found that $\mathrm{HbA1c}$ levels correlated with the severity of coronary atherosclerosis in both diabetic and non-diabetic patients. $\mathrm{HbA} 1 \mathrm{c}$ levels were the only independent predictor of severe CAD. In a recent meta-analysis, Liu et al. ${ }^{(28)}$ reported that elevated $\mathrm{HbA} 1 \mathrm{c}$ levels were an independent risk factor for mortality. In subgroup analyses, they found that an elevated $\mathrm{HbAlc}$ level predicted higher mortality risk in non-diabetics, while they did not detect an increased mortality risk in diabetics. Their conflicting findings between patients with and without diabetes as diabetic patients were tightly controlled in terms of risk factors such as dyslipidemias and hypertension.

The principal findings of Ashraf et al. (29) study indicated that HbAlc values, even in the normal range, are associated with the presence and severity of coronary lesions in people without diabetes, even after multivariable analysis models which was adjusted for traditional cardiovascular risk factors, insulin resistance and inflammatory markers. An increase in $\mathrm{HbA} 1 \mathrm{c}$ of one percent was associated with a 2.8-fold increase in CAD prevalence in the multivariate analysis and the best threshold of $\mathrm{HbA} 1 \mathrm{c}$ for prediction of the presence of CAD was 5.6\% (38 $\mathrm{mmol} / \mathrm{mol}$ ). The study also showed that glycemia in non-diabetic individuals, as a contributing factor for angiographic $\mathrm{CAD}$, might be more important in individuals with normal fasting glucose. The observation that $\mathrm{HbA} 1 \mathrm{c}$ desisted to be associated with the CAD in IFG group might be because of heavily clustered metabolic abnormalities in this group. In addition, hyperglycemia might enhance the proatherogenic effects of high sensitive (hs) CRP. Several findings suggest that hs-CRP might be related to atherogenesis by diminishing nitric oxide Verma et al. ${ }^{(30)}$. Interestingly, acute hyperglycemia also reduces nitric oxide bioavailability Giugliano et al. (31) , pointing to the possibility that the combined effect of raised hs-CRP concentrations and increased $\mathrm{HbA1c}$ could jointly reduce nitric oxide bioavailability.

Dutta et al. ${ }^{(32)}$ found that increasing HbA1c level was strongly correlated with disease severity, number of diseased vessels and higher SYNTAX score, in a graded fashion $(p<0.001)$ in non-diabetics. HbA1c values significantly correlated with the SYNTAX score. With increasing HbA1c levels, a significant increase was noted in the mean number of diseased vessels $(p<0.001)$. Similarly there was a linear correlation between $\mathrm{HbAlc}$ and number of vessels involved $(\mathrm{p}<0.001)$ in non-diabetics. Ghaffari et $\boldsymbol{a l}^{(\mathbf{3 3})}$ studied coronary angiographic findings and multivessel involvement based on HbAlc quartiles in non-diabetics. Patients with HbAlc $>5.8 \%$ had twice more chance of having triple-vessel diseases $(p=0.002)$ in non-diabetics. Kowalska et al. ${ }^{(34)}$ in a cohort of non-diabetic men referred for coronary angiography, demonstrated that the number of diseased vessels significantly correlated with increasing levels of HbAlc.

In the current study, we found a significant positive correlation between extent and severity of diseased vessels by CCT with total cholesterol, triglycerides, LDL-cholesterol, CAC score. Meanwhile, there was a significant negative correlation between extent and severity of diseased vessels by CCT with LVEF.

These results were concordant with Conkbayir et al. ${ }^{(35)}$ who revealed a significant relationship between lipid quartiles with the extent and severity of CAD. They also demonstrated significant roles of the TG/HDL-C ratio in predicting CAD risk among Turkish Cypriots.

\section{Study limitations:}

Small sample size of the studied patients and limited number of female patients included in the study. We did not have invasive angiographic data 
from our patients to further describe and confirming CAD severity. The selected group were referred for evaluation of suspected CAD; thus, this study may be affected by selection bias.

\section{CONCLUSIONS}

CAD was more advanced in prediabetic patients compared to non-diabetic patients. Moreover, nondiabetic levels of glucose metabolism evidenced by impaired FBG and increased levels of $\mathrm{HbA1C}$ were helpful to predict increased risk for the extent and severity of CAD among our study population.

\section{REFERENCES}

1. Scicali R, Giral P, Gallo A et al. (2016): $\mathrm{HbAlc}$ increase is associated with higher coronary and peripheral atherosclerotic burden in non diabetic patients. Atherosclerosis, 255:102-8.

2. Borch-Johnsen K, Neil A, Balkau B et al. (1999): Glucose tolerance and mortality: comparison of WHO and American Diabetes Association diagnostic criteria. Lancet, 354:617-21.

3. Zhu Y, Zhang C (2016): Prevalence of Gestational Diabetes and Risk of Progression to Type 2 Diabetes: a Global Perspective. Curr Diab Rep., 16:7-9.

4. Rivera JJ, Choi E-K, Yoon YE et al. (2010): Association between increasing levels of hemoglobin A1c and coronary atherosclerosis in asymptomatic individuals without diabetes mellitus. Coron Artery Dis., 21:157-63.

5. Greenland P, LaBree L, Azen SP et al. (2004): Coronary artery calcium score combined with Framingham score for risk prediction in asymptomatic individuals. JAMA., 291:210-5.

6. Detrano R, Guerci AD, Carr JJ et al. (2008): Coronary calcium as a predictor of coronary events in four racial or ethnic groups. N Engl J Med., 358:1336-45.

7. Goff David C, Lloyd-Jones M, Glen B et al. (2014): 2013 ACC/AHA Guideline on the Assessment of Cardiovascular Risk. Circulation, 129:S49-73.

8. Greenland P, Alpert JS, Beller GA et al. (2010): 2010 ACCF/AHA guideline for assessment of cardiovascular risk in asymptomatic adults: a report of the American College of Cardiology Foundation/American Heart Association Task Force on Practice Guidelines. J Am Coll Cardiol., 56:50103.

9. Schroeder S, Kuettner A, Beck T et al. (2005): Usefulness of noninvasive MSCT coronary angiography as first-line imaging technique in patients with chest pain: initial clinical experience. Int J Cardiol., 102:469-75.

10. American Diabetes Association (2010): Diagnosis and classification of diabetes mellitus. Diabetes Care, 1:62-69.

11. Haffner SM, Stern MP, Hazuda HP et al. (1990): Cardiovascular risk factors in confirmed prediabetic individuals. Does the clock for coronary heart disease start ticking before the onset of clinical diabetes? JAMA., 263:2893-8.

12. Moebus S, Stang A, Mohlenkamp S et al. (2009): Association of impaired fasting glucose and coronary artery calcification as a marker of subclinical atherosclerosis in a population-based cohort--results of the Heinz Nixdorf Recall Study. Diabetologia , 52:81-9.

13. Balkau B, Shipley M, Jarrett RJ et al. (1998): High blood glucose concentration is a risk factor for mortality in middle-aged nondiabetic men. 20-year follow-up in the Whitehall Study, the Paris Prospective Study, and the Helsinki Policemen Study. Diabetes Care, 21:360-7.

14. Berry C, Noble S, Gregoire JC et al. (2010): Glycaemic status influences the nature and severity of coronary artery disease. Diabetologia, 53:652-8.

15. Naito R, Miyauchi K, Ogita $M$ et al. (2014): Impact of admission glycemia and glycosylated hemoglobin A1c on long-term clinical outcomes of non-diabetic patients with acute coronary syndrome. J Cardiol., 63:106-11.

16. Lim S, Choi SH, Choi E-K et al. (2009): Comprehensive evaluation of coronary arteries by multidetector-row cardiac computed tomography according to the glucose level of asymptomatic individuals. Atherosclerosis, 205:156-62.

17. Garg N, Moorthy N, Kapoor A et al. (2014): Hemoglobin $\mathrm{A}(1 \mathrm{c})$ in nondiabetic patients: an independent predictor of coronary artery disease and its severity. Mayo Clin Proc., 89:908-16.

18. Kurihara O, Takano $M$, Seino $Y$ et al. (2015): Coronary atherosclerosis is already ongoing in prediabetic status: Insight from intravascular imaging modalities. World J Diabetes, 6:184-191.

19. Kurihara O, Takano M, Yamamoto $M$ et al. (2013): Impact of prediabetic status on coronary atherosclerosis: a multivessel angioscopic study. Diabetes Care, 36:729-33.

20. Ertan C, Ozeke O, Gul M et al. (2014): Association of prediabetes with diffuse coronary narrowing and small-vessel disease. J Cardiol., 63:29-34.

21. Gui MH, Li X, Lu ZQ et al. (2013): Fasting plasma glucose correlates with angiographic coronary artery disease prevalence and severity in Chinese patients without known diabetes. Acta Diabetol., 50:333-40.

22. Coutinho M, Gerstein HC, Wang Y et al. (1999): The relationship between glucose and incident cardiovascular events. A metaregression analysis of published data from 20 studies of 95,783 individuals followed for 12.4 years. Diabetes Care, 22:233-40.

23. Yan Q, Gu WQ, Hong J et al. (2009): Coronary angiographic studies of impaired glucose regulation and coronary artery disease in Chinese nondiabetic subjects. Endocrine, 36:457-63.

24. Tomizawa N, Inoh S, Nojo $T$ et al. (2016): The association of hemoglobin A1c and high risk plaque and plaque extent assessed by coronary computed tomography angiography. Int J Cardiovasc Imaging, 32:493-500.

25. Selvin E, Steffes MW, Zhu H et al. (2010): Glycated hemoglobin, diabetes, and cardiovascular risk in 
nondiabetic adults. N Engl J Med., 362:800-11.

26. Eskesen K, Jensen MT, Galatius $S$ et al. (2013): Glycated haemoglobin and the risk of cardiovascular disease, diabetes and all-cause mortality in the Copenhagen City Heart Study. J Intern Med., 273:94-101.

27. Ayhan SS, Tosun M, Ozturk S et al. (2012): Glycated haemoglobin is correlated with the severity of coronary artery disease independently of traditional risk factors in young patients. Endokrynol Pol., 63:367-71.

28. Liu Y, Yang Y, Zhu J et al. (2011): Prognostic significance of hemoglobin A1c level in patients hospitalized with coronary artery disease. A systematic review and meta-analysis. Cardiovasc Diabetol., 10:98-205.

29. Ashraf H, Boroumand MA, Amirzadegan A et al. (2013): Hemoglobin A1C in non-diabetic patients: an independent predictor of coronary artery disease and its severity. Diabetes Res Clin Pract., 102:225-32.

30. Verma S, Wang CH, Li SH et al. (2002): A selffulfilling prophecy: C-reactive protein attenuates nitric oxide production and inhibits angiogenesis. Circulation, 106:913-9.

31. Giugliano D, Marfella R, Coppola L et al. (1997):
Vascular effects of acute hyperglycemia in humans are reversed by L-arginine. Evidence for reduced availability of nitric oxide during hyperglycemia. Circulation, 95:1783-90.

32. Dutta B, Neginhal M, Iqbal F (2016): Glycated Hemoglobin (HbA1c) Correlation with Severity of Coronary Artery Disease in Non-diabetic Patients - A Hospital based Study from North-Eastern India. J Clin Diagn Res., 10:20-23.

33. Ghaffari S, Niafar F, Separham A et al. (2015): Association between HbA1c levels with severity of coronary artery disease and short-term outcomes of acute ST-elevation myocardial infarction in nondiabetic patients. Ther Adv Cardiovasc Dis., 9:305-13.

34. Kowalska I, Prokop J, Bachorzewska-Gajewska H et al. (2001): Disturbances of glucose metabolism in men referred for coronary arteriography. Postload glycemia as predictor for coronary atherosclerosis. Diabetes Care, 24:897-901.

35. Conkbayir C, Ayça B, Ökçün EB (2015): Lipid Variables Related to the Extent and Severity of Coronary Artery Disease in Non-Diabetic Turkish Cypriots. Iran J Public Health, 44:1196-203. 